\title{
Sprachwissenschaft
}

\author{
Anna Bajerowska \\ ORCID: 0000-0002-2894-8165
}

Universität Warschau, Warszawa

https://doi.org/10.19195/0435-5865.145.8

\section{Beitrag zur Theorie dynamischer Fachsprachen}

\begin{abstract}
s
Die vorliegende Arbeit thematisiert das Problem dynamischer Fachsprachen vor dem Hintergrund der europäischen Integration. Im ersten Teil wird der Versuch unternommen, das Wesen der europäischen Integration in Anlehnung an das von Franciszek Grucza vorgeschlagene Integrationskonzept zu erfassen. Im Mittelpunkt der Erwägungen steht die Annahme, dass die Europäische Union dem europäischen Integrationsprozess gegenüber ein sekundäres Gebilde darstellt. Im Fokus des zweiten Teils steht die Charakteristik ausgewählter Aspekte sog. dynamischer Fachsprachen. Es ist hervorzuheben, dass die hier präsentierten Überlegungen lediglich eine theoretische Grundlage für die künftige Erforschung kognitiver Funktionen dynamischer Fachsprachen darstellen.
\end{abstract}

Schlüsselwörter: Europäische Union, europäische Integration, dynamische Fachsprachen, terminologische Präzision, Innovationspotenzial, prognostisches Potenzial

\section{Contribution to the Theory of Dynamic Languages for special purposes}

The aim of this paper is to characterize Languages for Special Purposes designed and generated to describe the dynamic reality of European Integration and the European Union. First, the concept of European Integration will be outlined, which presents this phenomenon as an ongoing process taking place in EU-citizens' heads. The European Union is therefore perceived as its institutional emanation. Second, selected aspects of dynamic Languages for Special Purposes will be described. It has to be highlighted that the present considerations are preliminary and constitute merely a theoretical foundation for the future research on cognitive functions of dynamic Languages for Special Purposes.

Keywords: European Union, European integration, dynamic languages for special purposes, terminological precision, innovation potential, prognostic potential 
Anna Bajerowska, Uniwersytet Warszawski, Wydział Lingwistyki Stosowanej, ul. Szturmowa 4, p. 510,02-678 Warszawa, Polen, E-Mail: a.b.bajerowska@uw.edu.pl

Received: 30.09 .2019 , accepted: 8.04.2020

\section{Europäische Union und europäische Integration}

Eine tiefgreifende Reflexion über die mit der Europäischen Union eng verflochtenen Fachsprachen ist untrennbar mit dem Versuch verbunden, das Wesen der europäischen Integration und der Europäischen Union zu erfassen. Einen derartigen Versuch hat F. Grucza $(2002,2003,2004,2008 b)$ unternommen. Der Autor stellt fest, dass der Sinn der europäischen Integretion nicht vorrangig darin besteht, ein bestimmtes wirtschaftliches Entwicklungsniveau zu erreichen, sog. Zuschüsse zu erhalten oder das Wohlstandsniveau weniger wohlhabender Mitgliedstaaten zu steigern. Auch Argumente geopolitischer Natur weist F. Grucza zurück: die Möglichkeit, sich innerhalb der Europäischen Union frei bewegen zu können stelle keinen wesentlichen Aspekt der europäischen Integration dar. Das Wesen und das wichtigste Ziel der europäischen Integration bildet eine dauerhafte Sicherstellung von Frieden, die eine notwendige Voraussetzung für Wohlstand ist. Dauerhafter Frieden ist ausschließlich dadurch erreichbar, dass das Konfliktrisiko innerhalb und außerhalb der Europäischen Union gesenkt wird. Dies kann kein internationales Abkommen sichern. Ein durch Zusammenarbeit und Vertrauen erreichter Frieden kann ausschließlich durch die europäische Integration erreicht werden (s. F. Grucza 2008b: 37; Vgl. Ahlt, Szpunar 2001; Barzini 2001; Milczarek, Nowak 2003).

F. Grucza hebt hervor, dass eine immer tiefere Integration und das daraus resultierende Entstehen immer größerer Gemeinschaften, besonders vor dem Hintergrund der zivilisatorischen Entwicklung, als notwendig zu erachten ist. Der technologische Fortschritt konfrontiert die Menschen mit neuen Aufgaben und Herausforderungen, deren Bewältigung immer intensivere Bemühungen und immer mehr Aufwand in vielen Bereichen erfordert. Darüber hinaus geht der Fortschritt mit bisher unbekannten Gefahren einher, die auf der Ebene der einzelnen EU-Mitgliedstaaten, sowohl in quantitativer als auch in qualitativer Hinsicht, kaum eliminiert werden können. In einer breiteren zivilisatorischen Perspektive erscheint die europäische Integration als ein einzigartiges, notwendiges Mittel, um einen Raum (sowohl im sozialen als auch im geistig-kulturellen Ausmaß) harmonischen, gemeinschaftlichen Zusammenlebens zu schaffen.

Die europäische Integration ist nicht als ein andauernder Zustand, sondern eher als ein kontinuierlich fortschreitender Prozess anzusehen, woraus zu schlieBen ist, dass sie sich permanent in statu nascendi befindet (s. F. Grucza 2008b: 38; Vgl. Barzini 2001; Milczarek, Nowak 2003). Deswegen können die zu integrierenden Subjekte (Gemeinschaften oder Personen) entweder einander näher kom- 
men oder sich voneinander entfernenen (s. F. Grucza 2008b: 38; Vgl. Bajerowska 2014).

Demnach stellt die europäische Integration einen entweder fortschreitenden oder regressiven Prozess dar. Dieser kann aber unter keinen Umständen zu einer katatonischen Existenzform einer Gemeinschaft werden, weil er von Natur aus keinerlei Petrifizierungstendenz unterliegt. Auch F. Gołembski betrachtet die europäische Integration als einen zeitbedingten bzw. zeitgebundenen Prozess:

Die Integration - aufgefasst als ein Prozess - ist mit der Zeitlichkeit verbunden und vollzieht sich über die Zeit. Dies weist darauf hin, dass die Integration ein phasenhaft verlaufender Prozess ist. Seine einzelnen Phasen sind jeweils auf die vorgehenden Veränderungen beziehbar. Der Integrationsprozess wird dadurch iniziiert, dass im bisherigen, als stabil anzusehenden System sozialer Verhältnisse Veränderungen aufzutreten beginnen. [...] Ein jedes System wird durch einen bestimmten Komplex gegenseitiger Relationen zwischen den konstitutiven Systemkomponenten gekennzeichnet. Diese Relationen verleihen ihm einen dauerhaften Charakter und eine entsprechende Stabilität. Kommt es zu Veränderungen innerhalb dieses Relationennetzes, wird das gesamte System verändert. Der damit in Gang gesetzte Modifizierungsprozess resultiert darin, dass ein neues System entsteht. Ein derartig konzipierter Integrationsprozess basiert hauptsächlich darauf, in einem bereits existierenden System Veränderungen vorzunehmen, die zum Entstehen eines neuen Systems führen. [...] Die Integration bezieht sich in erster Linie auf den Zusammenhalt, d.h. auf einen Prozess, aus dem sich eine neue Komponentenstruktur ergeben soll." (Gołembski 2008: 22; Vgl. Milczarek, Nowak 2003; übers. von A.B. $)^{1}$

Der Autor betrachtet die Integration als einen Prozess, der verschiedene Modifizierungen innerhalb gegenseitiger Relationen der einzelnen „Komponenten” einer nicht näher bestimmten Kategorie voraussetzt. Die erwähnten Modifizierungen gehen jeweils mit einer Desintegration des existierenden Systems einher. Dies führt zur Entstehung eines Systems, das vollkommen neue Eigenschaften aufweist. Die Integration bedeutet demnach eine Transformation durch Desintegration von Relationen, die zwischen den Komponenten eines gegebenen Wirklichkeitsbereichs bestehen. Als solche vollzieht sie sich dadurch, dass die bestehende Wirklichkeit durch Modifizierungen innerhalb gegenseitiger Relationen ihrer konstitutiven Komponenten oder durch das Entstehen neuer Relationen verändert

1 „Integracja - ujmowana jako proces - odwołuje się do czasowości, dokonuje się w czasie. Oznacza to, że można wyróżnić jej poszczególne fazy, które odnoszą się do zachodzących zmian. Do inicjacji procesu integracji dochodzi w momencie, kiedy pojawiają się pierwsze zmiany w istniejącym dotychczas układzie stosunków społecznych, który można uznać za względnie trwały. [...] Każdy układ charakteryzuje się określonym stanem wzajemnych relacji zachodzących między współtworzącymi go elementami. To właśnie one nadają mu odpowiedni stan trwałości, stabilności. Jeżeli dojdzie do zmiany tych relacji, układ zaczyna podlegać zmianom. Rozpoczyna się proces przekształceń, które prowadzą do powstania nowego układu. Proces integracji rozpatrywany w tym kontekście oznacza przede wszystkim dokonywanie określonych zmian w istniejącym układzie, prowadzących do powstania nowego. [...] Integrowanie odnosi się w pierwszym znaczeniu do scalania, a więc do procesu, którego rezultatem powinien być nowy układ elementów." (Gołembski 2008: 22) 
wird. Ein anschauliches Beispiel hierfür stellt die Umgestaltung des nationalen Rechts durch die Umsetzung von EU-Richtlinien dar.

Es ist hervozuheben, dass sich der Integrationsprozess auf mindestens zwei Ebenen vollzieht: auf der gemeinschaftlichen Ebene (d.h. wenn die gesamte Gemeinschaft erwünschte Eigenschaften, Fertigkeiten erwirbt und sich bestimmte Denkweisen aneignet) und auf der Ebene von Individuen (z.B. bei den Kommunikationsakten zwischen den einzelnen Gemeinschaftsmitgliedern). Die erwähnten Ebenen durchdringen sich in besonderer Weise, wenn z.B. gewisse Gewohnheiten, Denkweisen, Aktivitäten auf der Ebene der gesamten Gemeinschaft verändert werden und wenn diese Veränderungen aus Prognosen über den Verlauf des europäischen Integrationsprozess resultieren (s. Bajerowska 2014). Unter Initiativen, die einen bedeutsamen Einfluss auf die Lebensweise zahlreicher sozialer Gruppen in ganz Europa ausgeübt haben, ist das Erasmus+ Programm, die Entwicklung des ESSnet-Kultur Modells, eines modularen statistischen Rahmenwerks für den Kultursektor sowie die Einrichtung des Schengen-Raums zu nennen.

Ein weiteres Problem, das beim Reflektieren über die (fach-)sprachlichen Aspekte der europäischen Integration thematisiert werden soll, ist ihre Reichweite, d.h. der Wirklichkeitsausschnitt, in dem sich der Integrationsprozess vollzieht. Der europäische Integrationsprozess vollzieht sich in den Gehirnen konkreter Menschen, vor allem in den Gehirnen der Bürger eines jeden EU-Mitgliedstaates, und zwar durch Internalisierung von Wissen bezüglich materieller, konzeptueller und geistig-kultureller Komponenten der europäischen Wirklichkeit. Hier handelt es sich um Wissen und Fähigkeiten, die im Laufe des Integrationsprozesses aufgrund einer relevanten Reflexion entstehen. Auf der gemeinschaftlichen Ebene ist die europäische Integration als Vereinigung oder Schnittmenge von Bestandteilen mentaler Wirklichkeit aufzufassen, die die in Betracht gezogenen Gemeinschaftsmitglieder im Integrationsprozess generieren.

Die Reichweite der europäischen Integration präzise zu bestimmen, stellt eine praktisch undurchführbare Aufgabe dar. Rein formell gesehen haben die Grenzen der europäischen Integration ihre Entsprechung bzw. Emanation im geopolitischen Ausmaß. Man könnte annehmen, dass sie sich mit den EU-Grenzen decken. Vor dem Hintergrund der oben dargelegten Erörterungen bleibt der Integrationsprozess in den EU-Kandidatenstaaten immer noch ein offenes Problem (Vgl. F. Grucza 2008b, Bajerowska 2014).

Demnach erfolgt die eigentliche Implementierung des europäischen Integrationsprozesses unabhängig von dem EU-Strukturrahmen, woraus zu schließen ist, dass die Europäische Union der europäischen Integration gegenüber ein sekundäres Gebilde darstellt. F. Grucza (2008b; Vgl. Ahlt, Szpunar 2001; Milczarek, Nowak 2003) stellt fest, dass die Europäische Union als Emanation der europäischen Integration und zugleich als ein wichtiges Mittel zu ihrer Verwirklichung anzusehen ist. Darüber hinaus soll hervorgehoben werden, dass die Europäische Union 
eine systematische bzw. regelmäßige Verwirklichung des Integrationsprozesses ermöglicht, und zwar nach Prognosen über die künftige Gestalt der gesamten Gemeinschaft.

Wie oben bereits angedeutet, befindet sich die Europäische Union als Emanation und institutionelles Fundament der europäischen Integration in statu nascendi, d.h. sie unterliegt kontinuierlichen Umwandlungen (s. F. Grucza 2008b: 39; Vgl. Milczarek, Nowak 2003). Aus diesem Grund ist sie als ein durchaus dynamisches Gebilde zu betrachten.

Sowohl die europäische Integration als auch die Europäische Union bilden „zukunftsorientierte” Phänomene (s. F. Grucza 2008b: 45), deren Zusammenspiel auf einem spezifischen „Rückkopplungseffekt” basiert. Die Europäische Union macht den europäischen Integrationsprozess im institutionellen Ausmaß möglich (s. F. Grucza 2008b: 40; Vgl. Brodecki 2011; Kenig-Witkowska, Łazowski, Ostrihansky 2011). Die primäre Funktion sowohl der Europäischen Union als auch der europäischen Integration besteht darin, bisher nicht existierende Wirklichkeitskomponenten zu generieren, die den gemeinsamen sozialen, geistig-kulturellen, wirtschaftlichen etc. Raum gestalten, und damit die Funktionsweise der europäischen Gemeinschaft in Einklang mit relevanten Prognosen umzugestalten. Ein treffendes Beispiel stellt hier die Einrichtung des Schengen-Raums als Reaktion auf eine voranschreitende Integration, Migration und die aus ihr resultierende Umwandlung des europäischen Arbeitsmarktes dar. Tiefgründige Modifikationen innerhalb von Wirklichkeitsbereichen, die das gemeinschaftliche Zusammenleben bestimmen, implizieren Veränderungen auf der Ebene relevanter Fachsprachen.

\section{Zu dynamischen Fachsprachen}

Eine entsprechende Qualität relevanter Fachsprachen, in denen die für die europäische Integration konstituierenden Faktoren beschrieben werden und in denen die „Transformationspläne” bezüglich der Letzteren entworfen werden, stellt eine Voraussetzung dafür dar, den Sinn und das Wesen der europäischen Integration zu erfassen sowie ihre zielgerechte Entwicklung zu gewährleisten. Aus diesem Grund hat die europäische Integration weitreichende Implikationen sprachlicher Natur (s. F. Grucza 2008b: 63; Vgl. Gołembski 2008; F. Grucza 2002, 2004; Schubert 2007). Dies ergibt sich vor allem aus der Tatsache, dass die Mitglieder der europäischen Gemeinschaft über Projekte kommunizieren, die neu entstehende Bestandteile der europäischen Wirklichkeit betreffen, sowie über unausweichliche Transformationen innerhalb der einzelnen nationalen Gemeinschaften, unter besonderer Berücksichtigung geistig-kultureller Komponenten ihrer Wirklichkeit.

Die gemeinschaftliche bzw. gesellschaftliche Bedeutung von Fachsprachen, dank denen der Prozess der europäischen Integration und seine Auswirkungen 
beschrieben werden, hängt von der Wichtigkeit der von ihnen denotierten Wirklichkeitsbereiche ab (s. F. Grucza 2008b: 35, 2003; Vgl. Schubert 2007). Die durch diese Sprachen denotierten Objekte, Zustände bzw. Phänomene bestimmen nicht nur die professionelle Tätigkeit der einzelnen Berufsgemeinschaften, sondern üben einen direkten Einfluss auf die Lebensqualität der gesamten Gemeinschaft aus. Von besonderem Belang ist hier die Fachsprache der europäischen Rechtsakte, in der z.B. Regelungen bezüglich des EU-Binnenmarkts formuliert werden.

Die Möglichkeit, den Prozess der europäischen Integration zielgerecht und effektiv zu fördern, hängt in hohem Maße mit der Qualität des Wissens über den Sinn und die Natur der europäischen Integration zusammen, das die Prozessteilnehmer internalisiert bzw. generiert haben (s. F. Grucza 2008b: 41; Vgl. Milczarek, Nowak 2003). Die Wissensqualität wäre demnach als Derivat relevanter Fachsprachkenntnise zu betrachten. Das Wissen über konstituierende Komponenten einer Gemeinschaft, emergiert durch den Erwerb relevanter (Fach-)Sprachen. Auf der Zeichenebene bilden die Letzteren eine gewisse Emanation dieser Komponenten. In Bezug darauf stellt F. Grucza fest, dass bestimmte Fachwissenspartien als Derivate der Kenntnisse entsprechender Fachsprachen zu betrachten sind (s. F. Grucza 2008b: 42).

Das Wissen bildet den Kern des sog. Innovationspotenzials und zugleich einen grundlegenden Faktor der Wettbewerbsfähigkeit. Der Kompetenzgrad im Bereich der Wissensgenerierung, -organisation und des Wissensmanagements bestimmt die Produktivität und die Wettbewerbsfähigkeit einzelner Wirtschaftsakteure und gesamter sozialer Gruppen (s. S. Grucza 2008: 115). Im Lichte der anthropozentrischen Fachsprachentheorie soll zwischen Innovationspotenzialen der Idioebene, d.h. Innovationspotenzialen konkreter Fachleute und Innovationspotenzialen der Gemeinschaftsebene unterschieden werden. Das gemeinschaftliche Innovationspotenzial kann als Schnittmenge dieser Eigenschaften aufgefasst werden. Ein derartig aufgefasstes Innovationspotenzial existiert in Form von konkreten Eigenschaften bzw. Attributen im Gehirn eines jeden in Betracht gezogenen Experten. Wird das Innovationspotenzial jedoch als Vereinigung bzw. logische Summe aufgefasst, d.h. unter Berücksichtigung aller Eigenschaften von Innovationspotenzialen der in Betracht gezogenen Experten, kann es nicht als ein wirkliches Seiendes betrachtet werden. Die Qualität derartig aufgefasster Innovationspotenziale hängt mit der Qualität ihrer Exponenten zusammen, d.h. mit der Qualität von Texten, die Innovationen im gegebenen Fachgebiet thematisieren. Die Qualität der Ausdrucksebene relevanter Texte ist ein wahrnehmbares Anzeichen für die Fähigkeit, (Fach-)Wissen zu generieren bzw. zu organisieren.

Wie bereits angedeutet, erfüllen Fachsprachen in erster Linie kognitive Funktionen. Im Fall von Fachsprachen, in denen die Europäische Union beschrieben wird, wird der kognitive Aspekt in besonderer Weise in den Vordergrund gerückt. Das Wissen über die europäische Integration, über ihre Grundlagen und ihren Sinn 
ist in hohem Ausmaß den Kenntnissen relevanter Fachsprachen zu verdanken. Es bestehen darüber hinaus tiefe Verflechtungen zwischen der Wissensqualität der Subjekte des europäischen Integrationsprozesses und der Qualität relevanter (Fach-)Sprachen sowie der Qualität relevanter (Fach-)Texte.

Fachsprachen, die gewisse für die europäische Integration und die Europäische Union konstituierende Komponenten denotieren, bilden demnach eine spezifische Kategorie von Sprachen, die ihre kognitive Funktion auf eine hoch spezifische Art und Weise erfüllen. Von der Qualität dieser Sprachen hängt die Wissensqualität der am europäischen Integrationsprozess beteiligten Subjekten ab. Mit der Qualität ihres Wissens geht wiederum die Qualität der von ihnen getroffenen Entscheidungen einher, wobei die Letzteren die entgültige Gestalt der europäischen Integration und damit das Schicksal der gesamten Gemeinschaft bestimmen.

Die mit der Europäischen Union eng verbundenen Fachsprachen denotieren Wirklichkeitsbereiche, die ständigen Umwandlungen unterliegen, d.h. dynamische Wirklichkeitsbereiche. Als solche werden sie als dynamische Fachsprachen betrachtet, d.h. als Sprachen, deren Bedeutungsfunktionen je nach Entwicklungsrichtung relevanter Wirklichkeitsausschnitte aktualisiert werden müssen (s. F. Grucza 2008b: 66 f.; Vgl. Aitchison 2003; Konerding 1993; Ziem 2008).

F. Grucza führt zwei fundamentale Gründe dafür an, die mit der Europäischen Union verbundenen Fachsprachen als dynamische Sprachen anzusehen. Erstens erfolgt aus einer jeden Modifizierung innerhalb des Zustands oder der Funktion der gegebenen Gemeinschaftswirklichkeit bzw. aus einem jeden Gesinnungswandel gegenüber ihren Komponenten ein gewisses Novum, sowohl auf der geistig-kulturellen bzw. sozialen als auch auf der wirtschaftlichen Ebene. Zweitens ruft eine jede Veränderung innerhalb des mit den erwähnten Wirklichkeitskomponenten verbundenen Wissens eine nötige Modifizierung von Bedeutungsfunktionen bestimmter lexikaler Einheiten relevanter (Fach-)Sprachen hervor (s. F. Grucza 2008b: 67; Vgl. Barzini 2001).

Im Zusammenhang damit, dass die hier besprochenen (Fach-)Sprachen hoch dynamische Wirklichkeitsbereiche denotieren, sollen sie Fragen nach der Spezifik künftiger Transformationen der betreffenden Wirklichkeitsausschnitte sowie nach ihrer künftigen Gestalt formulieren lassen. Sie sollen die Möglichkeit eröffnen, Aussagen über Prognosen und Projekte bezüglich der europäischen Integration und der Europäischen Union, vor allem aber bezüglich der „Transformationspläne” zu formulieren (s. F. Grucza 2008b: 50; Vgl. Barzini 2001). Aus den oben angeführten Gründen werden die mit der Europäischen Union verbundenen Sprachen von F. Grucza als projizierende Fachsprachen bezeichnet. Als solche sollen sie nicht nur diagnostisches und/oder anagnostisches, sondern auch prognostisches und applikatives Wissen ausdrücken (s. F. Grucza 2008b: 67).

Die Notwendigkeit, projizierenden Fachsprachen einen dynamischen Charakter zu verleihen sowie relevante Bedeutungsveränderungen dem Tempo anzupas- 
sen, dem in die Umwandlungen innerhalb ihres Denotationsbereichs vollzogen werden, führt dazu, dass ihre lexikalischen Einheiten normative Anforderungen sensu stricto kaum erfüllen können (s. F. Grucza 2008b: 67).

Wird die Präzision und Qualität der Zusammenhänge zwischen denotativen Komponenten und dem Wert der ihnen zugeordneten semantischen Bereiche nicht aktualisiert, werden die neu entstandenen Zustände unangemessen beurteilt, d.h. nach dem alten, nicht mehr aktuellen semantischen Wert relevanter Ausdrücke. Dies würde dazu führen, dass die Gemeinschaftsmitglieder neue Wirklichkeitskomponenten durch das Prisma von Überzeugungen und Urteilen wahrnehmen würden, die in Bezug auf nicht mehr existierende Zustände formuliert werden (s. F. Grucza 2008b: 68; Vgl. Barzini 2001).

Als ,wesentliche konstitutive Komponenten” (s. F. Grucza 2008b: 66) der einzelnen Fachwelten bestimmen Fachsprachen die Grenzbereiche der von ihnen denotierten Wirklichkeitsausschnitte. Im Fall von den mit der europäischen Integration und der Europäischen Union verbundenen Fachsprachen sind diese Grenzen besonders wandelbar und unscharf. Dies ergibt sich aus der Tatsache, dass a) die Grenzen zwischen den einzelnen Fachsprachlexiken und relevanten Allgemeinsprachen unscharf sind und sich jeglicher Konventionalisierung entziehen und dass b) die Grenzen zwischen den einzelnen Fachsprachen auch unscharf sind. Die hohe Wandelbarkeit der Grenzbereiche zwischen den Lexiken der einzelnen Sprachen, sowohl zwischen Fachsprachen und Allgemeinsprachen, als auch zwischen den einzelnen Fachsprachen, resultiert aus der Dynamik der durch diese Fachsprachen denotierten Wirklichkeitsbereiche. Dynamische Wirklichkeitsbereiche sind in besonderer Weise durch die „Migration” bestimmter Begriffe zwischen Fachsprachen verschiedener Fachwelten betroffen. Dies geht mit einer relativ hohen Zugänglichkeit gewisser für das gemeinschaftliche Zusammenleben wesentlicher Güter (materieller oder geistig-kultureller Natur) einher, z.B. werden medizinische Innovationen im sozial-wirtschaftlichen Diskurs angesprochen.

Wie oben bereits angedeutet, sollen im Fall von verschiedenen Fachsprachen, die die Konstituenten verschiedener Ausschnitte der Fachwirklichkeit denotieren, keine vereinheitlichten Methoden zur Erlangung terminologischer Präzision verwendet werden. Dies bezieht sich in erster Linie auf Fachsprachen, die dynamische Wirklichkeitsbereiche denotieren (s. F. Grucza 2008b: 66).

Für den Charakter der Fachsprachen, die die einzelnen Teilbereiche der Berufstätigkeit gewissermaßen semantisch bedienen, d.h. dafür, ob sie dynamischer oder projizierender Natur sind oder ob sie relativ stabile bzw. unveränderliche Objekte bzw. Zustände denotieren, sind teleologische Gründe entscheidend. Die auf ein und demselben Fachgebiet tätigen Mitglieder verschiedener Fachgemeinschaften können demnach Fachsprachen generieren, die durch einen unterschiedlichen Grad ihrer inneren Dynamik bzw. durch einen unterschiedlichen Entwicklungsgrad ihres prognostisch-projizierenden Potenzials gekennzeichnet sind (Vgl. Konerding 1993, Schubert 2007, Wendt 1997, Ziem 2008). 


\section{Fazit}

Die Idee, dynamische Fachsprachen den Gesetzen terminologischer Normung sensu stricto zu unterwerfen, würde eine zielgerechte Transferenz des diagnostischen, vor allen Dingen aber des prognostischen und projizierenden Wissens unmöglich machen. Anders ausgedrückt würde eine strikte Normalisierung dynamischer Fachsprachen den Weg zur Verwirklichung des europäischen Integrationsprozesses versperren, d.h. zur kognitiven Arbeit an den von ihnen denotierten Wirklichkeitsbereichen, in besonderer Weise zur Formulierung prognostischer und projizierender Aussagen über die konstitutiven Komponenten der europäischen Integration und der Europäischen Union. Im Zusammenhang damit soll die Beurteilung der Qualität dynamischer Fachsprachen nicht ausschließlich vom Präzisionsgrad ihrer Lexiken im terminologischen Sinne abhängig sein (s. F. Grucza 2008b: 69; Vgl. Wendt 1997).

Im Lichte der oben angeführten Erörterungen wird deutlich, dass die in den oben charakterisierten Fachsprachen formulierten Texte, die in Bezug auf besonders dynamische Wirklichkeitskomponenten verfasst werden, in prognostischer und projizierender Perspektive erforscht werden sollen. Die prognostische Perpektive muss in der linguistischen Forschung ihren Platz haben, in besonderer Weise bei den Bestimmungsversuchen des prognostisch-projizierendes Potenzials der zu untersuchenden Fachsprachen. Dies gilt sowohl in Bezug auf die Ausdrucksebene (die substanzielle Ebene) als auch auf die referentielle Ebene dynamischer Fachsprachen. Die Untersuchung des prognostisch-projizierenden Potenzials von Texten erfordert eine Analyse der Reichweite denotierter Wirklichkeitsbereiche sowie eine Bestimmung der Denotationsspezifik, die sich in der Adäquatheit der Ausdrucks- und Denotationsebene untersuchter Texte und in der Dynamik relevanter Referenzbereiche, d.h. der denotierten Wirklichkeitsbereiche manifestiert.

Auf der Ebene der linguistischen Modellierung wäre es höchstwahrscheinlich möglich, gewisse topologisch modellierbare Regelmäßigkeiten innerhalb der Dynamik (fach-)sprachlicher Umwandlungen auf intertextueller Ebene zu erfassen. Die Bestimmung der Topologie dynamischer sprachlicher Umwandlungen würde feststellen lassen, welche strukturellen Veränderungen die Entstehung neuer Wissenselemente begleiten, genauer gesagt, welche Relationen zwischen dem Emergieren neuer Wissenselemente und ihrer sprachlichen Exponenten bestehen, und zwar in einem derartigen System, das in stricte terminologischer Auffassung durch ein ungewöhnlich hohes Umwandlungstempo gestört wird.

Zusammenfassend ist festzustellen, dass dynamische Fachsprachen einen wichtigen Faktor sozialer Polarisierung darstellen. Von besonderem Belang ist F. Gruczas Verweis auf den Bedarf, die Verbreitung der Fachsprachenkenntnisse zu fördern, und zwar in Bezug auf solche Fachsprachen, die hoch dynamische Wirklichkeitsausschnitte wie die europäische Integration oder die Europäische 
Union denotieren (Vgl. F. Grucza 2008a: 19). Hinreichende Kenntnisse relevanter Fachsprachen stellen sowohl einen wichtigen Inklusionsfaktor als auch einen Exklusionsfaktor im sozialen Ausmaß dar.

\section{Literatur}

Ahlt, Michael / Szpunar, Maciej (2001): Prawo europejskie. Warszawa.

Aitchison, Jean (2003): Words in the Mind. An Introduction to the Mental Lexicon. Oxford.

Bajerowska, Anna (2014): Transferencja wiedzy specjalistycznej. Warszawa.

Barzini, Luigi (2001): Europejczycy. Warszawa.

Brodecki, Zdzisław (2011): Prawo integracji. Konstytucja dla Europy. Warszawa.

Gołembski, Franciszek (2008): Kulturowe aspekty integracji europejskiej. Warszawa.

Grucza, Franciszek (2002): Język (narodowy) - tożsamość (narodowa) - integracja (europejska). In: Jeleń, Elżbieta / Rauen, Monika / Świątek, Małgorzata / Winiarska, Justyna (Hrsg.): Zmiany i rozwój języka oraz tożsamości narodowej - trendy w procesie integracji europejskiej (Language Dynamics and Linguistic Identity in the Context of European Integration; Wandel und Entwicklung von Sprache und Identität - Tendenzen der europäischen Einigung). Kraków. S. 25-49.

Grucza, Franciszek (2003): Mehrsprachigkeit in Mitteleuropa und der Europäischen Union: Traditionen - Gefahren - Ausblicke. In: Besters-Dilger, Juliane / de Cillia, Rudolf / Krumm, Hans-Jürgen / Rindler-Schjerve, Rosita (Hrsg.): Mehrsprachigkeit in der erweiterten Europäischen Union. Klagenfurt. S. 15-27.

Grucza, Franciszek (2004): Integration - Wort und Phänomen. In: Hałub, Marek / Bartoszewicz, Iwona / Jurasz, Alina (Hrsg.): Werte und Wertungen. Sprach-, literatur- und kulturwissenschaftliche Skizzen und Schätzungen. Wrocław. S. 513-524.

Grucza, Franciszek (2008a): Języki specjalistyczne - indykatory i/lub determinanty rozwoju cywilizacyjnego. In: Lukszyn, Jerzy (Hrsg.): Podstawy technolingwistyki I. Warszawa. S. 5-23.

Grucza, Franciszek (2008b): O językach dotyczacych europejskiej integracji i Unii Europejskiej i potrzebie ukonstytuowania ogólnej lingwistyki języków specjalistycznych. In: Lukszyn, Jerzy (Hrsg.): Podstawy technolingwistyki I. Warszawa. S. 27-74.

Grucza, Sambor (2008): Lingwistyka języków specjalistycznych. Warszawa.

Kenig-Witkowska, Maria Magdalena / Łazowski, Adam / Ostrihansky, Rudolf (Hrsg.) (2011): Prawo instytucjonalne Unii Europejskiej. Warszawa.

Konerding, Klaus-Peter (1993): Frames und lexikalisches Bedeutungswissen. Untersuchungen zur linguistischen Grundlegung einer Frametheorie und zu ihrer Anwendung in der Lexikographie. Tübingen.

Milczarek, Dariusz / Nowak, Alojzy Z. (Hrsg.) (2003): Integracja europejska. Wybrane problemy. Warszawa.

Schubert, Klaus (2007): Wissen, Sprache, Medium, Arbeit. Ein integratives Modell der ein- und mehrsprachigen Fachkommunikation. Tübingen.

Wendt, Susanne (1997): Terminus - Thesaurus - Text. Theorie und Praxis von Fachbegriffsystemen und ihrer Repräsentation in Fachtexten. Tübingen.

Ziem, Alexander (2008): Frames und sprachliches Wissen. Kognitive Aspekte der semantischen Kompetenz. Berlin.

Germanica Wratislaviensia 145, 2020

(C) for this edition by CNS 